\title{
El Acuerdo Comercial de Relaciones Económicas más Estrechas entre Australia y Nueva Zelanda
}

\section{Introducción}

$\mathrm{E}$ 1 Acuerdo Comercial de Relaciones Económicas más Estrechas entre Australia y Nueva Zelanda (ANZCERTA, su sigla en inglés), también conocido como Acuerdo de Relaciones Económicas más Estrechas (CER, su sigla en inglés), es más que un acuerdo de libre comercio. Se trata de un acuerdo que cubre casi todos los aspectos de las relaciones económicas y comerciales entre Australia y Nueva Zelanda, pero que, además de establecer una zona de libre comercio entre ambas naciones, también incluye temas como los servicios, el transporte, el mercado laboral, la armonización de procedimientos administrativos y sanitarios, entre otros puntos que progresivamente se fueron incorporando.

En las dos décadas posteriores a la entrada en vigor del ANZCERTA en 1983, el comercio y la inversión bilateral entre Australia y Nueva Zelanda registraron aumentos significativos como resultado del alto grado de integración económica alcanzado entre los dos países tras el acuerdo. Cabe mencionar que el ANZCERTA es considerado actualmente como uno de

* Profesor investigador del Departamento de Estudios del Pacífico de la Universidad de Guadalajara e investigador del SNI. los acuerdos bilaterales de libre comercio y cooperación más amplios, eficientes y exitosos en el contexto internacional.

El presente trabajo tiene tres propósitos. El primero consiste en describir en términos generales las principales características del ANZCERTA, así como las modificaciones que experimentó posteriormente; el segundo se enfoca a resaltar la importancia y los beneficios que el acuerdo representa y ha traído a la economía de los dos países, y el tercero busca identificar los principales retos que este acuerdo enfrenta actualmente.

\section{Descripción general y antecedentes del ANZCERTA}

El ANZCERTA es hoy en día el instrumento más importante que rige el comercio de bienes y servicios entre Australia y Nueva Zelanda, y entró en vigor el primero de enero de 1983. El acuerdo fue diseñado inicialmente para crear un área de libre comercio de bienes y servicios entre los dos países, si bien los servicios se incorporaron en 1988, e incluye disposiciones relativas a la competencia, la contratación pública y medidas antidumping, entre otros aspectos. Sobre esta base, el comercio de todos los productos originarios de Australia y Nueva Zelanda quedó libre de aranceles, restricciones cuantitativas o licencias 
de importación, medidas antidumping y de salvaguardas. De igual forma, se eliminaron los subsidios e incentivos a las exportaciones en el comercio entre los dos países, así como otros impedimentos administrativos al comercio y los flujos de inversión, entre ellos los procedimientos de compras gubernamentales. ${ }^{1}$

$\mathrm{El}$ antecedente inmediato del ANZCERTA fue un conjunto de acuerdos parciales sobre el comercio que concluyeron con la firma, en agosto de 1963, del Acuerdo de Libre Comercio Australia-Nueva Zelanda (NAFTA, su sigla en inglés), que entraría en vigor en enero de 1966. El NAFTA fue un acuerdo diseñado esencialmente para reducir la protección de un pequeño número de productos, pero excluía algunos sectores clave donde la liberalización recíproca pudo haber tenido un impacto significativo, de ahí que sus efectos en el comercio bilateral hayan sido más bien limitados. Es importante mencionar que, aun cuando la tarifa promedio mostró un aumento durante la vigencia del acuerdo, se logró eliminar más del 80\% de las restricciones cuantitativas al comercio entre Australia y Nueva Zelanda. ${ }^{2}$

No obstante, la creación de un área de libre mercado bilateral con la puesta en marcha del NAFTA no estuvo exenta de problemas. Los principales inconvenientes del acuerdo consistían en que sólo consideraba una gama reducida de bienes; muchos productos fueron excluidos del acuerdo, incluso aquellos que podrían haberse beneficiado con un mercado más amplio; no se estableció con precisión un itinerario para la reducción de las tarifas; hubo intereses políticos de ambos lados que pugnaron por seguir protegiendo a la industria nacional existente; frecuentemente se recurría a comités consultivos cuando algún sector del comercio de alguno de los países se veía amenazado y, finalmente, el acuerdo no fue suficientemente consistente para eliminar las barreras al comercio.

Fue así como, dadas las carencias y limitaciones del NAFTA, en enero de 1983 se firmó el ANZCERTA o CER con la finalidad de contrarrestar las deficiencias del primer acuerdo y de ampliar las relaciones económicas entre Australia y Nueva Zelanda. ${ }^{3}$ En este sentido, los objetivos del nuevo acuerdo fueron los siguientes:

a) Procurar una relación más amplia entre Australia y Nueva Zelanda.

b) Desarrollar relaciones económicas más estrechas mediante una expansión mutuamente benéfica del libre comercio entre Australia y Nueva Zelanda.

c) Eliminar las barreras al comercio entre ambos países de manera gradual y progresiva bajo un calendario de desgravación y con un mínimo de desajustes.

d) Desarrollar el comercio entre Australia y Nueva Zelanda bajo condiciones de competencia limpia.

De esta forma, con el ANZCERTA se estableció el marco necesario para alcanzar en forma progresiva un área de libre comercio mucho más efectiva y dinámica entre ambos países. Con este nuevo acuerdo se buscaba una sustancial liberalización del comercio, en la medida en que se fijaron límites a las restricciones cuantitativas y se acordó un calendario para la eliminación gradual de tarifas, cuotas y subsidios a la exportación; no obstante, su alcance se vería aún limitado por un simple hecho: todos los productos se incluían, excepto los explícitamente excluidos de la negociación, además de los servicios.

Bajo estas circunstancias, el ANZCERTA fue renegociado en 1988 mediante la firma de tres protocolos:

1. El protocolo sobre la aceleración del libre comercio de bienes. Este protocolo 
permitió adelantar el plazo propuesto para la disminución de los aranceles y las restricciones cuantitativas en vigencia para el comercio de bienes para finales de 1990.

2. El protocolo sobre armonización de procedimientos administrativos y sanitarios. Con este protocolo se eliminó el uso de medidas antidumping en el comercio bilateral y de subsidios a industrias específicas que afectaran la competencia, se amplió el alcance de la no-discriminación en compras gubernamentales $\mathrm{y}$ se armonizaron las políticas regulatorias, incluyendo aquellas leyes de competencia que afectaran el comercio de bienes.

3. El protocolo sobre el comercio de servicios. Este protocolo permitió incorporar los servicios al acuerdo, lo que trajo consigo el establecimiento de un sistema de reglas transparentes, la liberalización de las barreras al comercio de los servicios y el aumento de la competencia.

Aún así, esta renegociación continuó excluyendo del acuerdo algunos sectores, como las telecomunicaciones, la radiodifusión, el transporte aéreo, el transporte marítimo de cabotaje y el servicio de correos. Adicionalmente, a propuesta de Australia, se incluyeron algunas restricciones en el sector bancario y ciertas preferencias ha- cia empresas australianas de ingeniería, construcción y consultoría. Esta serie de excepciones se estableció en forma temporal, por lo que se fueron eliminando paulatinamente.

Una nueva revisión del ANZCERTA, realizada en 1992, permitiría eliminar gran parte de las restricciones australianas en el sector de servicios. Por su parte, Nueva Zelanda eliminó las reservas sobre radio y televisión, difusión de onda corta y satelital, y parte de las reservas sobre aeronavegación. Cabe mencionar, que ambos países conservaron su posición respecto de las telecomunicaciones y el servicio de correos, pero acordaron trabajar de manera conjunta en la integración de los mercados nacionales de aeronavegación. ${ }^{4}$ Del mismo modo, se comprometieron a dar tratamiento nacional a las empresas de ambos países, tratamiento de nación más favorecida en los sectores no incluidos en el acuerdo y acceso al mercado para los servicios cubiertos. No obstante, la no mención de las regulaciones no discriminatorias, es decir, las restricciones cuantitativas, como impedimento para el acceso al mercado, implicaba que dichas restricciones podían ser mantenidas. ${ }^{5}$

Con respecto a la mano de obra, como en el caso de la Unión Europea, Australia y Nueva Zelanda forman un mercado común de trabajo desde 1920. Por tanto, los ciudadanos de cada uno de los países son libres de trabajar en ambos. Asimismo, han 
acordado leyes de competencia similares y no se aplican procedimientos antidumping al comercio en la zona. De igual forma, el acuerdo prevé la futura incorporación de otros países, previa negociación conjunta entre el potencial integrante y los actuales miembros.

\section{Principales beneficios del ANZCERTA}

Sin duda alguna, las dos economías se han visto beneficiadas en términos de la competencia, el mejoramiento de los sistemas de producción y de comercialización, la ampliación de la cooperación y el entendimiento mutuo, todo ello a partir del acuerdo. Esto ha sido posible gracias a que el ANZCERTA constituye un mecanismo activo de integración económica que es revisado periódicamente mediante reuniones anuales realizadas entre los ministros de comercio de Australia y Nueva Zelanda, en las cuales se discuten diversos aspectos relativos al comercio y la cooperación de ambos países para mejorar su efectividad.

Dada la naturaleza misma del acuerdo, uno de sus beneficios más notables es el observado en el sector externo de los dos países en general y, de manera particular, en la relación comercial entre ambos, donde el comercio bilateral experimentó importantes cambios a partir del acuerdo. ${ }^{6}$ Como se puede apreciar en el cuadro 1, las exportaciones de Australia y Nueva Zelanda mostraron un fuerte impulso, ya que mientras en la primera mitad de los años ochenta apenas lograron un leve crecimiento, para la segunda mitad registraron un incremento superior al 50\% en cada caso. Posteriormente, se observa un brusco descenso en su comportamiento para finales de los años noventa, como consecuencia de la caída de los mercados a escala internacional y, particularmente, de los de la región por los efectos de la crisis financiera que afectó a la mayor parte de las economías asiáticas. Es a partir del año 2000 cuando nuevamente las exportaciones de los dos países muestran una significativa recuperación, especialmente en el caso de Nueva Zelanda (cuadro1).

En cuanto a las importaciones, ellas también manifestaron un fuerte impulso para finales de los años ochenta, más aún en el caso de Australia, donde aumentaron casi el 75\%; no obstante, al igual que las exportaciones, aunque en menor proporción, tendieron a la baja para fines de los años noventa. Cabe destacar que, a partir del año 2000, las importaciones de ambos países volvieron a mostrar un considerable incremento, incluso por arriba del mostrado por las exportaciones, y fue mayor en el caso de Nueva Zelanda. Lo anterior sugiere que estas dos economías se encuentran altamente integradas al mercado internacional, por lo que el comportamiento de su sector externo depende en gran medida de los vaivenes del mercado internacional, pero también depende del tamaño de sus

\section{Cuadro 1}

Comercio exterior de Australia y Nueva Zelanda (crecimiento durante el periodo en por ciento)

\begin{tabular}{|c|c|c|c|c|}
\hline \multirow[t]{2}{*}{ Periodo } & \multicolumn{2}{|c|}{$\begin{array}{l}\text { Exportaciones } \\
\text { totales }\end{array}$} & \multicolumn{2}{|c|}{$\begin{array}{l}\text { Importaciones } \\
\text { totales }\end{array}$} \\
\hline & Australia & $\begin{array}{c}N . \\
\text { Zelanda }\end{array}$ & Australia & $\begin{array}{c}\text { N. } \\
\text { Zelanda }\end{array}$ \\
\hline 1980-1984 & 5.32 & 1.79 & 15.71 & 13.34 \\
\hline 1985-1989 & 64.24 & 55.16 & 73.56 & 46.6 \\
\hline 1990-1994 & 19.56 & 29.71 & 27.25 & 25.39 \\
\hline 1995-1999 & 5.59 & -8.72 & 12.85 & 2.45 \\
\hline $2000-2004$ & 35.31 & 53.5 & 52.91 & 66.84 \\
\hline \multicolumn{5}{|c|}{$\begin{array}{l}\text { Fuente: Elaborado con base en "Statistics } \\
\text { Database", WTO. }\end{array}$} \\
\hline
\end{tabular}

MÉXICO YLACUENCADELPACÍFICO vol. 8, núm. 25 / mayo - agosto de 2005 
respectivas economías y del tamaño y la conformación de su sector externo. ${ }^{7}$

Con respecto al comercio bilateral, éste cobró un fuerte impulso con la entrada en vigor del ANZCERTA, de manera particular durante los años ochenta y la primera mitad de los años noventa. En el periodo 1980-1990, el comercio total de mercancías entre los dos países creció el $12 \%$ en promedio por año, en tanto que para el primer lustro de los años noventa aumentó poco más del $14 \%$ en promedio por año. Posteriormente, el comercio total bilateral presentó una brusca caída en su ritmo de crecimiento, hasta ubicarse en alrededor del $3 \%$ en promedio por año para el primer lustro de la presente década, si bien este descenso en el intercambio de mercancías afectó más a Nueva Zelanda, debido a que concentra alrededor de una cuarta parte de su comercio con Australia, mientras que este otro país presenta una estructura más diversificada en su relación comercial con el exterior (cuadro 2).

\section{Cuadro 2}

Comercio bilateral entre Australia y Nueva Zelanda (valores en por ciento para el periodo)

\begin{tabular}{lc}
\hline Periodo & $\begin{array}{c}\text { Crecimiento del comercio } \\
\text { bilateral }\end{array}$ \\
\hline $1980-1984$ & 73.1 \\
$1985-1989$ & 61.8 \\
$1990-1994$ & 77.8 \\
$1995-1999$ & 20.8 \\
$2000-2005$ & 15.1 \\
\hline
\end{tabular}

Fuente: Elaborado con base en Department of Foreign Affairs and Trade,"Direction of Trade Time Series. One Hundred Years of Trade", Australia, February 2002; Statistics New Zealand, "New Zealand External Trade Statistics", June 2005.

Australia se ha convertido en el principal socio comercial de Nueva Zelanda en términos de exportaciones e importaciones de bienes y servicios. Y, en forma paralela, Australia representa la principal fuente y el principal destino de la inversión extranjera directa de Nueva Zelanda. Para el año 2005, Australia era el principal destino de las exportaciones de Nueva Zelanda, al alcanzar $20 \%$ del total de las exportaciones neozelandesas. Dichas exportaciones han aumentado en promedio $9 \%$ por año desde 1999, y en ellas sobresalen básicamente aceites, oro, madera, queso, maquinaria y aparatos eléctricos, animales vivos, aeronaves y artículos de plástico. Del lado de las importaciones de Nueva Zelanda, el $22 \%$ del total proviene de Australia, y observaron un crecimiento del 11\% en promedio por año desde 1999; entre ellas, sobresalen combustibles, vehículos de motor, oxido de aluminio, medicamentos y aparatos electrónicos. ${ }^{8}$ Por el contrario, Nueva Zelanda tiene una menor importancia como mercado para Australia, ya que participa con menos del 7\% de las exportaciones totales y el $4 \%$ del total de las importaciones de Australia (cuadro 3).

Otro de los beneficios tangibles del ANZCERTA es el concerniente al rubro de la inversión extranjera directa. Cabe señalar que la inversión extranjera bilateral ha aumentado alrededor del 8\% en promedio por año desde 1999. Actualmente, alrededor del 55\% del total de la inversión directa de Nueva Zelanda tiene como destino Australia, mientras que el $36 \%$ de la inversión extranjera directa en Nueva Zelanda proviene de Australia. ${ }^{9}$ De la misma forma, la proximidad entre los dos países representa un factor clave para el turismo. Los australianos constituyen el principal mercado de origen de turistas para Nueva Zelanda, ya que contribuyen con el 16\% de los ingresos totales por concepto de turismo de este último, en tanto Australia es el principal 


\section{Cuadro 3}

Comercio bilateral entre Australia y Nueva Zelanda (participación en el total de cada país en por cientos)

\begin{tabular}{ccccc}
\hline Año & \multicolumn{2}{c}{$\begin{array}{c}\text { Australia con Nueva } \\
\text { Zelanda }\end{array}$} & $\begin{array}{r}\text { Nueva Zelanda con } \\
\text { Australia }\end{array}$ \\
\hline & $\begin{array}{c}\text { Exporta- } \\
\text { ciones }\end{array}$ & $\begin{array}{c}\text { Importa- } \\
\text { ciones }\end{array}$ & $\begin{array}{c}\text { Exporta- } \\
\text { ciones }\end{array}$ & $\begin{array}{c}\text { Importa- } \\
\text { ciones }\end{array}$ \\
\hline 1980 & 4.7 & 3.4 & 12.0 & 21.0 \\
1985 & 4.6 & 4.2 & 15.5 & 17.0 \\
1990 & 4.9 & 4.4 & 18.1 & 20.3 \\
1995 & 7.4 & 4.6 & 18.7 & 21.5 \\
2000 & 5.6 & 3.9 & 20.8 & 23.4 \\
2005 & 6.6 & 3.5 & 20.1 & 22.2 \\
\hline
\end{tabular}

Fuente: Elaborado con base en Department of Foreign Affairs and Trade, "Direction of Trade Time Series. One Hundred Years of Trade", Australia, February 2002; Australian Bureau of Statistics, "International Trade in Goods and Services", Australia, November 2005; Elizabeth Petersen, "Closer economic relations between Australia and New Zealand: specialization, competitiveness complementarity", Economic Society of Australia, 2003; Statistics New Zealand, "New Zealand External Trade Statistics", New Zealand, June 2005.

destino turístico para los neozelandeses, ya que más de la mitad del total de turistas de Nueva Zelanda se dirigen a ese país.

\section{Temas pendientes y retos del ANZCERTA}

Entre los principales temas pendientes que enfrenta actualmente el ANZCERTA están algunas restricciones que permanecen en la relación comercial entre Australia y Nueva Zelanda, particularmente en el sector de las telecomunicaciones y de la banca, que desde un principio quedaron excluidos del acuerdo. Otros temas pendientes por resolver tienen que ver con problemas de doble tributación que enfrentan ciertos accionistas de ambos países; de igual forma, no existen disposiciones específicas para el libre movimiento de capitales, aunque se han logrado importantes avances en la armonización de sus respectivas legisla- ciones para fomentar la competencia y los negocios. ${ }^{10}$

Pero quizá el reto más significativo del ANZCERTA consiste en recuperar el dinamismo en el intercambio comercial bilateral alcanzado durante los años ochenta y noventa, situación que se vuelve cada vez más difícil. Por un lado, Australia ha estado concentrando sus relaciones comerciales cada vez más con los países del Este de Asia, de manera particular con Japón, China, Corea del Sur, Singapur y Taiwan, así como con Estados Unidos. Por otro lado, por el tamaño de su economía, Nueva Zelanda representa un mercado muy limitado y, al parecer, cada vez menos atractivo para Australia. ${ }^{11}$

Resulta claro que Nueva Zelanda, al ser una economía más pequeña que la australiana, se ha visto beneficiada en mayor medida con el ANZCERTA en relación con Australia en términos del comercio y la inversión. Pero, por otro lado, Australia se ha estado integrando mejor y diversificando más sus relaciones comerciales con los países del Este de Asia, debido probablemente al mayor tamaño de su economía y a su mejor desempeño económico, lo que posiblemente explica el paulatino debilitamiento en la relación comercial entre ambos países. my

\section{Notas}

1 Sobre la base de: Elizabeth Petersen (2003), "Closer Economic Relations between Australia and New Zealand: Specialization, Competitiveness, Complementarity", Economic Society of Australia.

2 De acuerdo con cifras de Gustavo Girado, "Australia y su vinculación con Argentina. Características del ANZCERTA vis a vis el MERCOSUR", Secretaría de Industria, Comercio y Minería del Ministerio de Economía y Obras y Servicios Públicos, Argentina, 1998.

3 Para un conocimiento más detallado del acuerdo véase, Department of Foreign Affairs and Tra- 
de, "Australia New Zealand Closer Economic Relations Trade Agreement", Canberra, Australia, marzo de 1995.

4 A partir de noviembre de 1994, las empresas de aeronavegación australianas y neozelandesas pueden cubrir rutas locales o internacionales en ambos países.

$5 \quad$ G. Girado, op. cit., 1998.

6 Es importante destacar que la puesta en marcha del ANZCERTA se da en el contexto de las reformas económicas emprendidas en Australia en 1983 y Nueva Zelanda en 1984. Dichas reformas incluyeron la liberalización del comercio, la remoción de los controles sobre los precios y el tipo de cambio, la eliminación de los subsidios a la producción y al comercio, la desregulación del sistema financiero, cambios en la política fiscal, la privatización de empresas estatales y nuevas leyes sobre la competencia, entre otras medidas. De ahí que es muy difícil precisar en qué grado el crecimiento de las exportaciones de los dos países se debe exclusivamente al acuerdo o a las reformas económicas en general. Lo más probable es que ambos factores hayan incidido de manera simultánea en este sentido.

7 Para el año 2003, el PIB de Australia ascendía a alrededor de 560 mil millones de dólares, lo que representaba casi 6.3 veces el tamaño del de Nueva Zelanda. Para el año 2004, el comercio total (exportaciones más importaciones) de Australia sumó 196 mil millones de dólares, esto es, 4.5 veces el de Nueva Zelanda (oecD, Country Statistical Profiles 2005 y wTO, Statistical Database).

8 Ídem.

9 De acuerdo con datos de Jason Attewell, "Australia is New Zealand's most Significant Trading Partner", International Accounts Division of Statistics New Zealand, noviembre de 2002: http://www.stats.govt.nz

10 Véase, Sheila Page, "Regionalism among Developing Countries", Nueva York, Palgrave Publishers, 2000.

11 En 1980, Nueva Zelanda ocupaba el tercer lugar entre los principales mercados de exportación de Australia. Para el año 2000, había descendido al quinto lugar. Ver Department of Foreign Affaire and Trade, op. cit., febrero de 2002.

\section{Bibliografía}

Attewell, Jason (2002), "Australia is New Zealand's most significant trading partner", International Accounts Division of Statistics New Zealand, noviembre, http://www.stats.govt.nz.

Australian Bureau of Statistics (2005), "International Trade in Goods and Services", Australia, noviembre, http://www.abs.govt.au.

Department of Foreign Affairs and Trade (1995), "Australia New Zealand Closer Economic Relations Trade Agreement", Canberra, Australia, marzo.

Department of Foreign Affairs and Trade (2002), "Direction of Trade Time Series. One Hundred Years of Trade", Australia, febrero.

Girado, Gustavo (1998), "Australia y su vinculación con Argentina. Características del ANZCERTA vis a vis el Mercosur", Argentina, Secretaría de Industria, Comercio y Minería del Ministerio de Economía y Obras y Servicios Públicos.

OECD (2005), "Country Statistical Profiles 2005", Francia, http://www.oecd.org.

Page, Sheila (2000), "Regionalism among Developing Countries" , Nueva York, Palgrave Publishers.

Petersen, Elizabeth (2003), "Closer Economic Relations between Australia and New Zealand: Specialization, Competitiveness, Complementarity", Economic Society of Australia.

- Statistics New Zealand (2005), “New Zealand External Trade Statistics", Nueva Zelanda, junio, http:// www.stats.govt.nz.

wTO, "Statistical Database", http://www.stat.wto.org. 